\title{
Statistical methods on detecting differentially expressed genes for RNA-seq data
}

\author{
Zhongxue Chen ${ }^{*}$, Jianzhong Liu ${ }^{2}$, Hon Keung Tony $\mathrm{Ng}^{3}$, Saralees Nadarajah ${ }^{4}$, Howard L Kaufman ${ }^{5,6}$, Jack Y Yang ${ }^{7}$, \\ Youping Deng ${ }^{5,8^{*}}$
}

From BIOCOMP 2010 - The 2010 International Conference on Bioinformatics and Computational Biology Las Vegas, NV, USA. 12-15 July 2010

\begin{abstract}
Background: For RNA-seq data, the aggregated counts of the short reads from the same gene is used to approximate the gene expression level. The count data can be modelled as samples from Poisson distributions with possible different parameters. To detect differentially expressed genes under two situations, statistical methods for detecting the difference of two Poisson means are used. When the expression level of a gene is low, i.e., the number of count is small, it is usually more difficult to detect the mean differences, and therefore statistical methods which are more powerful for low expression level are particularly desirable. In statistical literature, several methods have been proposed to compare two Poisson means (rates). In this paper, we compare these methods by using simulated and real RNA-seq data.
\end{abstract}

Results: Through simulation study and real data analysis, we find that the Wald test with the data being logtransformed is more powerful than other methods, including the likelihood ratio test, which has similar power as the variance stabilizing transformation test; both are more powerful than the conditional exact test and Fisher exact test.

Conclusions: When the count data in RNA-seq can be reasonably modelled as Poisson distribution, the Wald-Log test is more powerful and should be used to detect the differentially expressed genes.

\section{Background}

Recent advancements in deep sequencing technique enable the ultra-high-throughput sequencing (called the second- or next- generation sequencing) approaches, to be used for transcriptome, including gene expression, analyses [1-4]. RNA-seq produces a count (the total number of short reads which are annotated to positions within a gene or transcript) for each gene expression level. Some researchers have shown that the count data can be quite reasonably modelled as Poisson distribution $[1,3]$. To detect differentially expressed genes (DEGs) under two conditions (e.g., disease vs. healthy status),

\footnotetext{
* Correspondence: Zhongxue.Chen@uth.tmc.edu; youping_deng@rush.edu 'Biostatistics Epidemiology Research Design Core, Center for Clinical and Translational Sciences, The University of Texas Health Science Center at Houston, Houston, TX 77030, USA

${ }^{5}$ Rush University Cancer Center, Rush University Medical Center, Chicago, IL 60612, USA

Full list of author information is available at the end of the article
}

some statistical methods have been used. For example, Marioni et al [3] have proposed to use likelihood ratio test (LRT) based on a generalized linear model (GLM) with dependent variable (count) having Poisson distribution to detect DEGs under two conditions without biological replicates. Fisher exact test has also been proposed to detect DEGs for RNA-seq data $[5,6]$. Some researchers modelled the count data as binomial distribution $\operatorname{bin}(\mathrm{n}, \mathrm{p})$, where $\mathrm{n}$ is the total counts on the same lane, which usually ranges from several millions to tens of millions [7]. However, it is well known that for a binomial distribution bin(n,p) with large $n$ and small $p$, it can be approximated by Poisson distribution. It is expected that for RNA-seq data methods based on binomial distribution should have similar performance as those methods based on Poisson distribution and therefore are not considered in this paper.
C Biomed Central

(c) 2011 Chen et al. This is an open access article distributed under the terms of the Creative Commons Attribution License (http:// creativecommons.org/licenses/by/2.0), which permits unrestricted use, distribution, and reproduction in any medium, provided the original work is properly cited. 
If there are biological replicates, the count data may have larger variances than expected from the Poisson distribution as biological replicates bring extra variances. Under this kind of situations, Poisson distribution with over-dispersed variances or negative binomial distributions are thought to be more appropriate [8,9]. However, due to the high cost, recent studies rarely used biological replicates; we therefore focus on the situations where at most technical replicates are used and Poisson distribution is assumed to be appropriate.

Besides Fisher exact test and LRT from GLM, several other test approaches in statistical literature have been proposed to detect the difference of two Poisson means. For example, under the null hypothesis that $X_{1}$ and $X_{2}$ are both from $\operatorname{Poi}(\lambda)$, then given $k=X_{1}+X_{2}, X_{1}$ has a binomial distribution $\operatorname{Bin}(k, p)$, where $p=X_{1} / k$. Therefore the conditional exact test can be used to detect the difference of the two Poisson means. One of the advantages of the GLM is that it can incorporate some covariates that we are interested in. If no covariates are considered due to the small sample size and large number of genes, the LRT can be constructed directly. Similar to LRT, Wald test also has an asymptotical Chisquare distribution with $\mathrm{df}=1$ [10-12]. A modified Wald test (Wald-Log) with data being first log-transformed has also been proposed for detecting the difference between two Poisson means [11]. Another method called variance stabilizing transformation (VST) was proposed by Huffman $[10,13]$.

In this paper, we first compare the above mentioned methods by using simulations. Since for those methods having the same asymptotic distribution, they are expected to have similar performances for large sample sizes (e.g., Poisson distributions with large means), we focus on simulating Poisson distributions with small or moderate means. We then use a real RNA-seq data to show the performances of those methods.

\section{Results}

\section{Simulation results}

In the simulations, we first assume there are no replicates, i.e., each of the two conditions has only one sample. We assume the data for condition 1 is from a Poisson distribution with fixed mean 5, 10, 15, or 30 . For condition 2, we assume the Poisson mean is the same as or greater than that for condition 1 . We use the nominal significance level $10^{-3}$ to reflect the situation where in a study with a large number of significant variable (genes), a stringent p-value is needed. The estimated size and power are calculated from 10,000 runs for each setting.

Table 1 reports the estimated size and power for different situations. Figure 1 plots the estimated size and power for each method from this simulation.
Table 1 Estimated size and power (no replicates for each condition).

\begin{tabular}{|c|c|c|c|c|c|c|c|}
\hline Lambda1 & Lambda2 & LRT & Cond & Wald & $\begin{array}{l}\text { Wald- } \\
\text { Log }\end{array}$ & VST & Fisher \\
\hline \multirow[t]{6}{*}{5} & 5 & 0.0128 & 0.0002 & 0.0002 & 0.0185 & 0.0011 & 0.0002 \\
\hline & 9 & 0.0199 & 0.0066 & 0.0074 & 0.0522 & 0.0176 & 0.0066 \\
\hline & 13 & 0.0842 & 0.0492 & 0.0550 & 0.1737 & 0.0916 & 0.0492 \\
\hline & 17 & 0.2484 & 0.1877 & 0.1964 & 0.3976 & 0.2626 & 0.1877 \\
\hline & 21 & 0.4903 & 0.4248 & 0.4358 & 0.6269 & 0.4981 & 0.4248 \\
\hline & 25 & 0.7146 & 0.6547 & 0.6685 & 0.8077 & 0.7168 & 0.6547 \\
\hline \multirow[t]{7}{*}{10} & 10 & 0.0013 & 0.0004 & 0.0005 & 0.0041 & 0.0013 & 0.0004 \\
\hline & 15 & 0.0114 & 0.0082 & 0.0089 & 0.0256 & 0.0124 & 0.0082 \\
\hline & 20 & 0.0729 & 0.0504 & 0.0571 & 0.1190 & 0.0737 & 0.0504 \\
\hline & 25 & 0.2433 & 0.1863 & 0.2110 & 0.3217 & 0.2435 & 0.1863 \\
\hline & 30 & 0.4848 & 0.4111 & 0.4460 & 0.5746 & 0.4848 & 0.4111 \\
\hline & 35 & 0.7177 & 0.6625 & 0.6900 & 0.7859 & 0.7177 & 0.6625 \\
\hline & 40 & 0.8750 & 0.8414 & 0.8599 & 0.9102 & 0.8750 & 0.8414 \\
\hline \multirow[t]{8}{*}{15} & 15 & 0.0012 & 0.0007 & 0.0009 & 0.0030 & 0.0012 & 0.0007 \\
\hline & 20 & 0.0075 & 0.0047 & 0.0052 & 0.0140 & 0.0075 & 0.0047 \\
\hline & 25 & 0.0456 & 0.0314 & 0.0377 & 0.0689 & 0.0456 & 0.0314 \\
\hline & 30 & 0.1483 & 0.1130 & 0.1305 & 0.1990 & 0.1483 & 0.1130 \\
\hline & 35 & 0.3422 & 0.2868 & 0.3167 & 0.4121 & 0.3422 & 0.2868 \\
\hline & 40 & 0.5692 & 0.5046 & 0.5459 & 0.6266 & 0.5692 & 0.5046 \\
\hline & 45 & 0.7563 & 0.7047 & 0.7393 & 0.8002 & 0.7563 & 0.7047 \\
\hline & 50 & 0.8907 & 0.8569 & 0.8796 & 0.9096 & 0.8907 & 0.8569 \\
\hline \multirow[t]{6}{*}{30} & 30 & 0.0011 & 0.0007 & 0.0011 & 0.0019 & 0.0011 & 0.0007 \\
\hline & 40 & 0.0180 & 0.0125 & 0.0163 & 0.0224 & 0.0180 & 0.0125 \\
\hline & 50 & 0.1464 & 0.1167 & 0.1370 & 0.1704 & 0.1464 & 0.1167 \\
\hline & 60 & 0.4776 & 0.4292 & 0.4620 & 0.5109 & 0.4777 & 0.4292 \\
\hline & 70 & 0.7840 & 0.7526 & 0.7750 & 0.8145 & 0.7848 & 0.7526 \\
\hline & 80 & 0.9421 & 0.9299 & 0.9390 & 0.9514 & 0.9423 & 0.9299 \\
\hline
\end{tabular}

Estimated size and power from each method for detecting the difference of two Poisson means. There are no replicates for each condition. The Poisson means for the first condition are $5,10,15$, or 30 . The second Poisson means are the same as (for size) or larger than (for power) the first ones. The nominal size (significance level) is $10^{-3}$ and 10,000 runs are used for each setting.

In the second simulation, we assume there are three replicates for each condition. Table 2 shows the estimated sizes and powers and these results are plotted in Figure 2.

From the results of the two simulation studies, we can see that the Wald-Log method is usually more powerful than the LRT, which has similar power as that of VST; both are slightly better than the Wald test, which in turn outperforms Fisher test and conditional binomial test. It is noticeable that both Fisher and conditional tests are exact conditional tests and have almost identical powers.

\section{Detecting DEGs for a real RNA-seq dataset}

We also apply these statistical methods to a real RNAseq data generated by Illumina's sequencing technology [3]. In this dataset, we consider two different samples: 

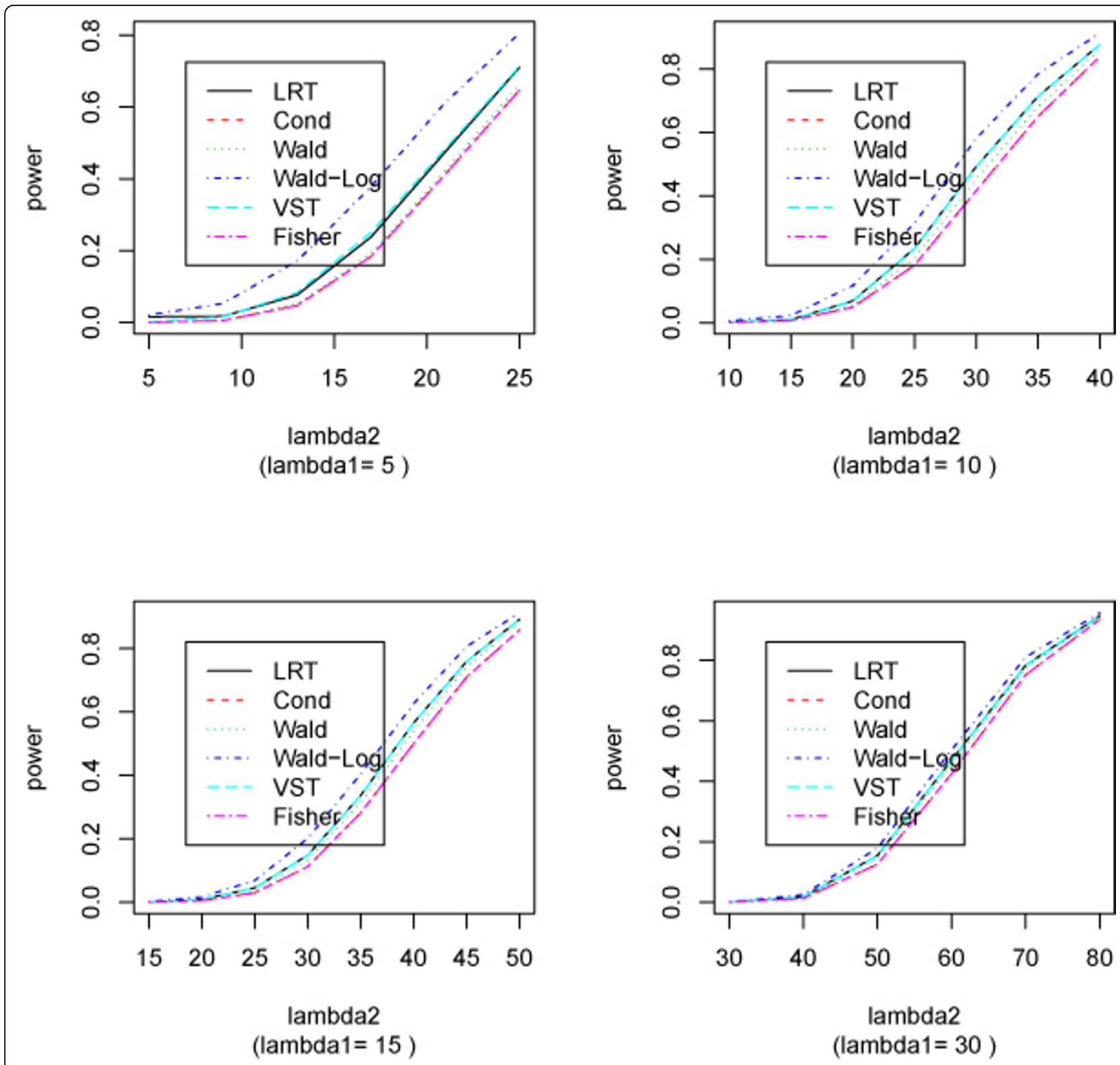

Figure 1 Estimated power from various methods (no replicates). Estimated size and power from each method for detecting the difference of two Poisson means. There are no replicates for each condition. The Poisson means for the first condition are 5, 10, 15, or 30. The second Poisson means are the same as (for size) or larger than (for power) the first ones. The nominal size (significance level) is $10^{-3}$ and 10,000 runs are used for each setting.

kidney and liver both at concentration 3PM, each has 5 lanes (technical replicates). We want to detect DEGs between these two samples. There are 15227 genes with at least 5 counts in total (i.e., on average 1 count on each lane) for both samples. For each of the 15227 genes, we calculate the $\mathrm{p}$-values using various methods mentioned above. Due to the almost identical performance between the Fisher and conditional binomial tests, we choose not to use the latter one in this real data analysis. Figure 3 plots the p-values from other methods compared with the LRT. For many genes, Wald-Log produces smaller p-values than the LRT does. We are particularly interested in seeing how these statistical methods perform for low expression data. To this purpose, we use the data from genes with average count per lane between 1 and 10. There are 2657 such genes from the data. Figure 4 plots the p-values (on - $\log 10$ scale) obtained from other methods compared with those from LRT for those 2657 genes. LRT, Fisher and VST obtain very similar p-values for those genes, 
Table 2 Estimated size and power (each condition has three replicates).

\begin{tabular}{llllllll}
\hline Lambda1 & Lambda1 & LRT & Cond & Wald & $\begin{array}{l}\text { Wald- } \\
\text { Log }\end{array}$ & VST & Fisher \\
\hline 5 & 5 & 0.0012 & 0.0009 & 0.0009 & 0.0027 & 0.0012 & 0.0009 \\
& 7 & 0.0102 & 0.0062 & 0.0077 & 0.0175 & 0.0103 & 0.0062 \\
& 9 & 0.0786 & 0.0542 & 0.0652 & 0.1150 & 0.0786 & 0.0542 \\
& 11 & 0.2517 & 0.2008 & 0.2265 & 0.3160 & 0.2517 & 0.2008 \\
& 13 & 0.5204 & 0.4581 & 0.4940 & 0.5872 & 0.5204 & 0.4581 \\
\hline 10 & 10 & 0.0008 & 0.0002 & 0.0006 & 0.0016 & 0.0008 & 0.0002 \\
& 12 & 0.0045 & 0.0029 & 0.0042 & 0.0065 & 0.0045 & 0.0029 \\
& 14 & 0.0316 & 0.0229 & 0.0279 & 0.0389 & 0.0316 & 0.0229 \\
& 16 & 0.1100 & 0.0882 & 0.1019 & 0.1272 & 0.1100 & 0.0882 \\
& 18 & 0.2602 & 0.2198 & 0.2454 & 0.2883 & 0.2603 & 0.2198 \\
& 20 & 0.4670 & 0.4210 & 0.4515 & 0.5005 & 0.4672 & 0.4210 \\
\hline 15 & 15 & 0.0007 & 0.0003 & 0.0007 & 0.0007 & 0.0007 & 0.0003 \\
& 17 & 0.0034 & 0.0026 & 0.0031 & 0.0049 & 0.0034 & 0.0026 \\
& 19 & 0.0181 & 0.0144 & 0.0172 & 0.0221 & 0.0182 & 0.0144 \\
& 21 & 0.0612 & 0.0505 & 0.0583 & 0.0712 & 0.0618 & 0.0505 \\
& 23 & 0.1442 & 0.1251 & 0.1395 & 0.1648 & 0.1460 & 0.1251 \\
& 25 & 0.2850 & 0.2564 & 0.2759 & 0.3125 & 0.2869 & 0.2564 \\
\hline 30 & 30 & 0.0014 & 0.0010 & 0.0013 & 0.0016 & 0.0014 & 0.0010 \\
& 34 & 0.0074 & 0.0057 & 0.0071 & 0.0082 & 0.0074 & 0.0057 \\
& 38 & 0.0520 & 0.0457 & 0.0505 & 0.0570 & 0.0520 & 0.0457 \\
& 42 & 0.2073 & 0.1846 & 0.2034 & 0.2210 & 0.2073 & 0.1846 \\
& 46 & 0.4543 & 0.4270 & 0.4486 & 0.4701 & 0.4543 & 0.4270 \\
\hline & 15 & & & & & &
\end{tabular}

Estimated size and power from each method for detecting the difference of means between two Poisson distributions. There are three replicates for each condition. The Poisson means for the first condition are 5, 10, 15, or 30 . The second Poisson means are the same as (for size) or larger than (for power) the first ones. The nominal size (significance level) is $10^{-3}$ and 10,000 runs are used for each setting.

while Wald produces larger p-values than LRT for a small portion of those genes. However, for many genes with small $\mathrm{p}$-values from LRT, their p-values obtained by Wald-log are smaller. This indicates the Wald-Log test is more powerful than LRT and other tests for this data.

To see how statistical significance (p-values) related to biological significance (fold changes), we plot the pvalues (on - $\log 10$ scale) vs. absolute fold change (on $\log 2$ scale) in Figure 5 from different methods for those 2657 genes. It can be seen that the Wald-Log test obtains smaller p-values than other methods when the fold changes are large, indicating this method can detecting more biologically meaningful DEGs than other methods for low expression genes.

Figure 6 plots the counts under two conditions for each low expression genes selected by Wald-Log and LRT at significance level $10^{-3}$. Of the 581 selected genes by Wald-Log, 498 are also selected by LRT. Each of the selected gene has at least 20 ounts in total for at least one sample (liver or kidney). The fold changes (liver vs. kidney, or kidney vs. liver) of all the 581 selected genes are all at least 2 . For those 83 genes selected by WaldLog but not by LRT, some even have fold changes as large as 4 .

Table 3 lists the numbers of DEGs obtained with different cutoff values by various methods. Consistent with what we have observed, the Wald-Log test identifies more DEGs than other methods for low expression genes. This is also true when we apply these statistical tests to all of the 15227 genes.

\section{Discussion}

Some studies have shown that for RNA-seq data without biological replicates, the count data can be reasonably modelled as Poisson distributed [1,3]. Since it is usually more difficult to detect DEGs with low expression level for RNA-seq data [14], it is desirable to find powerful statistical tests for detecting low expression DEGs. Through simulations and real data, we show that the Wald-Log is more powerful that the commonly used LRT and Fisher exact test. The test statistic of WaldLog has an asymptotic normal distribution. Like many other test statistics having asymptotic normal or Chisquare distributions, the asymptotic distributional results for the Wald-Log test statistic do not hold when the sample sizes are small (i.e., the Poisson mean is small). However, from our simulations we can see that when the Poisson mean is greater than 10, it can have reasonable sizes even if there are no replicates.

It is interesting to see that the Wald-Log test is also related to the fold changes (see method section for more details); therefore it is also more biologically meaningful. The test statistic of Wald-Log not only uses the fold change (on log scale), but also considers the variances associated with FC. For low expression level, the FC has larger variances, indicating it is more difficult to identify DEGs for low expression genes. This is consistent with the observation from other studies $[14,15]$ : the ability to detect DEGs is strongly associated with the length of gene as longer genes potentially have more aggregated tag counts.

The denominator of the Wald-Log test statistic is: $\sqrt{\left(2+n_{1} / n_{2}+n_{2} / n_{1}\right) /\left(\sum_{j=1}^{n_{1}} X_{1 j}+\sum_{j=1}^{n_{2}} X_{2 j}\right)}$, which is the estimated standard error of the fold change (log scale). If we change the square root to: $\left[\left(2+n_{1} / n_{2}+n_{2} / n_{1}\right) /\left(\sum_{j=1}^{n_{1}} X_{1 j}+\sum_{j=1}^{n_{2}} X_{2 j}\right)\right]^{1 / k}$, then when $\mathrm{k}=$ 2 , it is the usual Wald-Log test; when $k \rightarrow \infty$, it becomes the fold change criterion as the denominator equals to 1. When the count data has an over-dispersed Poisson distribution, the above estimator underestimates the 

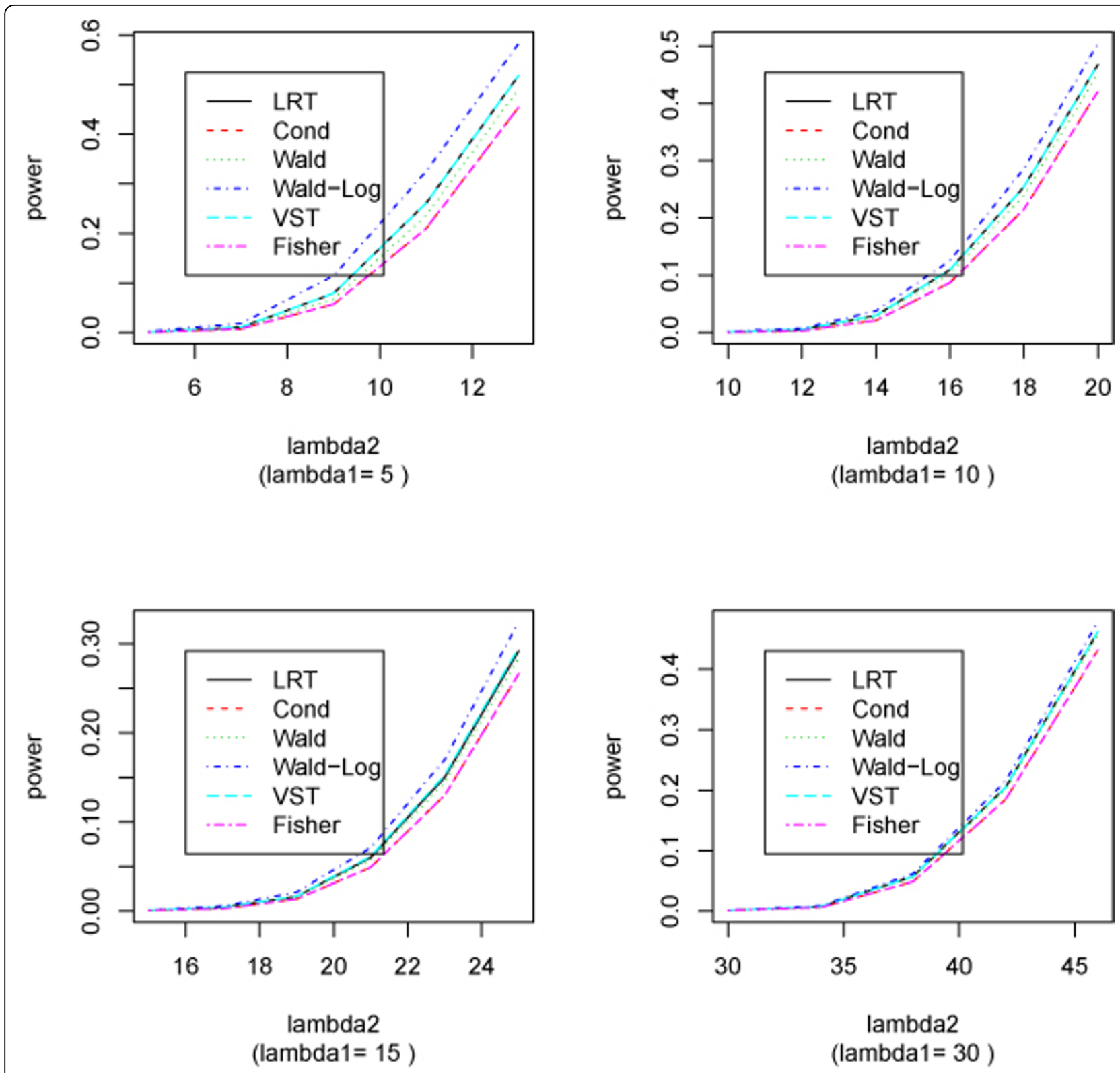

Figure 2 Estimated power from various methods (with 3 replicates for each condition). Estimated size and power from each method for detecting the difference of two Poisson means. There are three replicates for each condition. The Poisson means for the first condition are 5, 10, 15, or 30. The second Poisson means are the same as (for size) or larger than (for power) the first ones. The nominal size (significance level) is $10^{-3}$ and 10,000 runs are used for each setting.

standard error and we will have inflated type I error rate. Under this situation, we need to derive a new estimator for the standard error; or a Bayesian approach assuming the standard error follows a specific distribution can be built. It should be noticed that for the Bayesian approaches, choosing appropriate prior distributions are critical and may have huge impacts on the results. We may also choose models other than the Poisson model. For example, if there are biological replicates, the count data may have over-dispersed variances, other models such as over-dispersed Poisson distribution or negative binomial distribution can be used $[8,9]$.

\section{Conclusions}

When detecting the difference of two Poisson means, if the Poisson means are large, all the statistical tests mentioned above have very similar power. However, their 


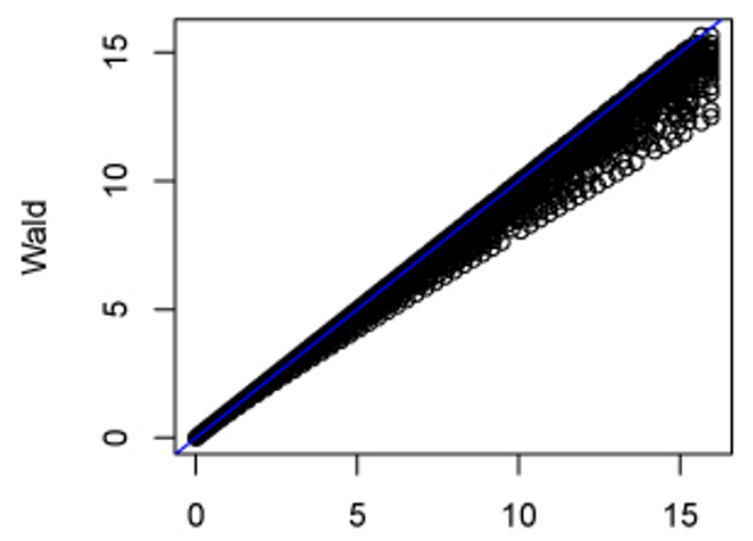

LRT

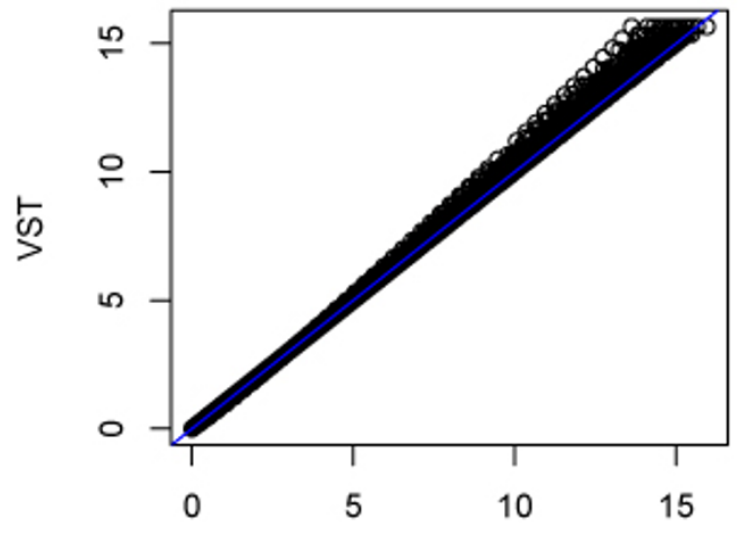

LRT

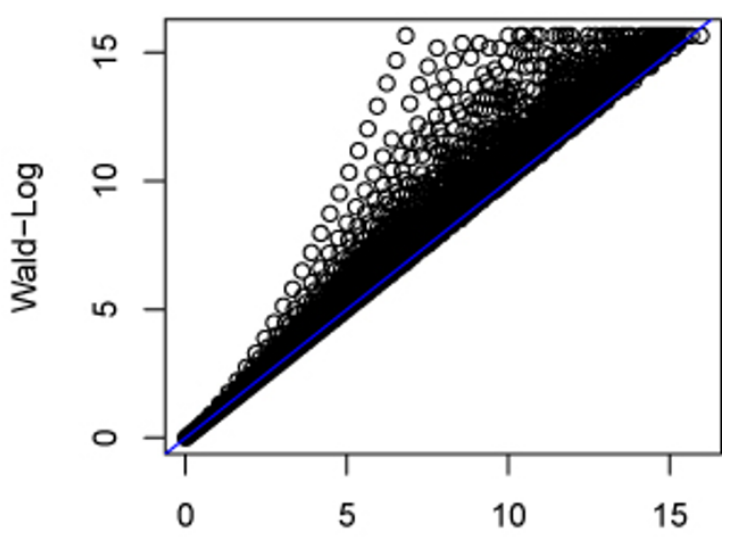

LRT

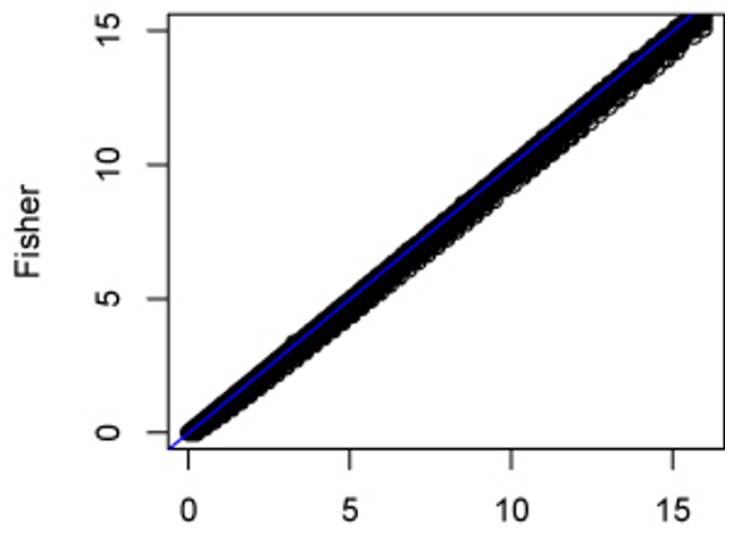

LRT

Figure 3 P-values for the real RNA-seq data from different methods. Compare p-values (-log10 scale) obtained from other methods with those from LRT for the 15227 genes whose average counts per lane are at least one for each sample.

powers may differ a lot when the Poisson means are small. When the count data in RNA-seq can be reasonably modelled as Poisson distribution, it is desirable to choose a statistical test which outperforms others for low expression genes. Through simulation study and real RNA-seq data analysis, we have shown that the Wald-Log test is more attractive than other methods and should be used to identify DEGs.

\section{Methods}

Likelihood ratio test (LRT)

Suppose there are $\mathrm{n}_{1}$ and $\mathrm{n}_{2}$ technical replicates for condition 1 and condition 2, respectively; denote the count of short reads of a gene of the $j^{\text {th }}$ replicate under condition $i$ by $\mathrm{x}_{\mathrm{ij}}$, where $\mathrm{i}=1,2, \mathrm{j}=1,2, \ldots, \mathrm{n}_{\mathrm{i}}$. We also assume random variables $\mathrm{X}_{\mathrm{ij}} \sim \operatorname{Poi}\left(\lambda_{i}\right)$.

The test statistic of LRT is:

$$
\begin{gathered}
T_{L R T}=2\left[\left(\sum_{j=1}^{n_{1}} X_{1 j}\right) \log \left(\frac{\hat{\lambda}_{1}}{\hat{\lambda}_{0}}\right)+\left(\sum_{j=1}^{n_{2}} X_{2 j}\right) \log \left(\frac{\hat{\lambda}_{2}}{\hat{\lambda}_{0}}\right)+n_{1}\left(\hat{\lambda}_{0}-\hat{\lambda}_{1}\right)+n_{2}\left(\hat{\lambda}_{0}-\hat{\lambda}_{2}\right)\right], \\
\text { where } \hat{\lambda}_{0}=\frac{\sum_{j=1}^{n_{1}} X_{1 j}+\sum_{j=1}^{n_{2}} X_{2 j}}{n_{1}+n_{2}}, \quad \hat{\lambda}_{1}=\frac{\sum_{j=1}^{n_{1}} X_{1 j}}{n_{1}}, \quad \hat{\lambda}_{2}=\frac{\sum_{j=1}^{n_{2}} X_{2 j}}{n_{2}} .
\end{gathered}
$$

Under the null hypothesis that the two Poisson means are the same, the test statistic has an asymptotic Chisquare distribution with degree of freedom (df) equals to 1 . 


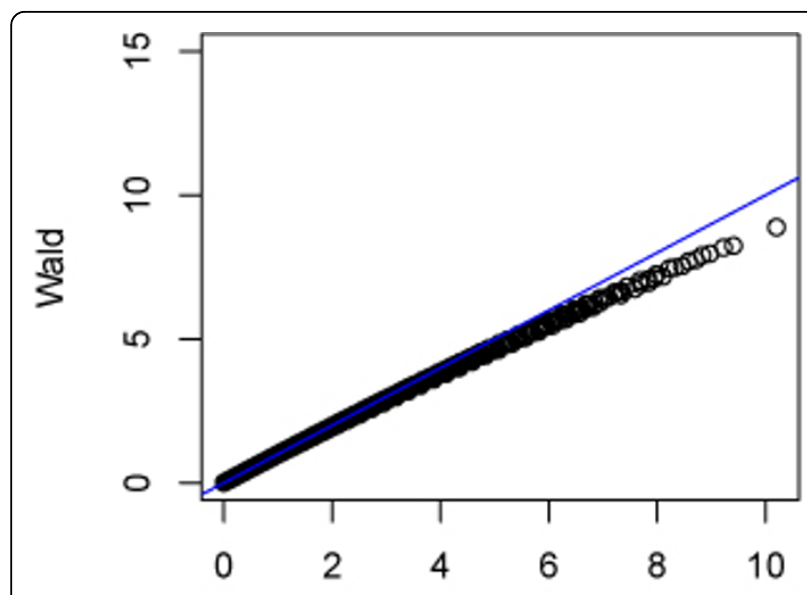

LRT

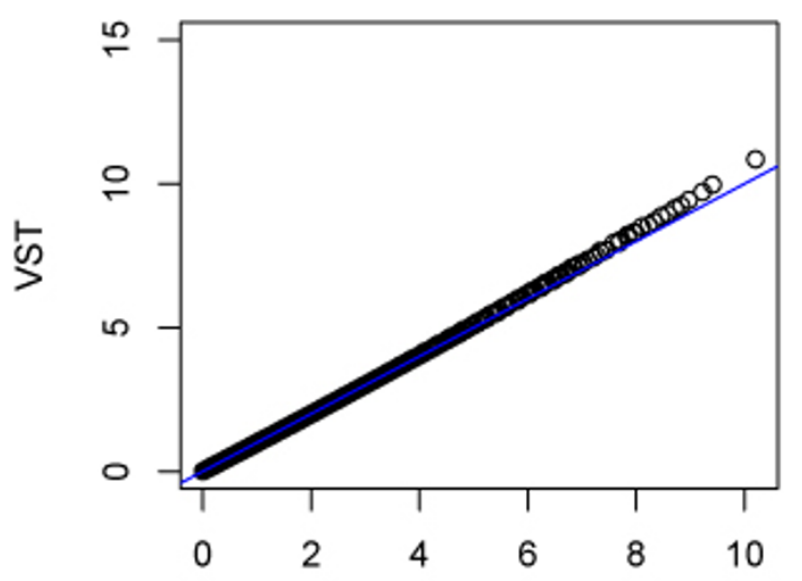

LRT

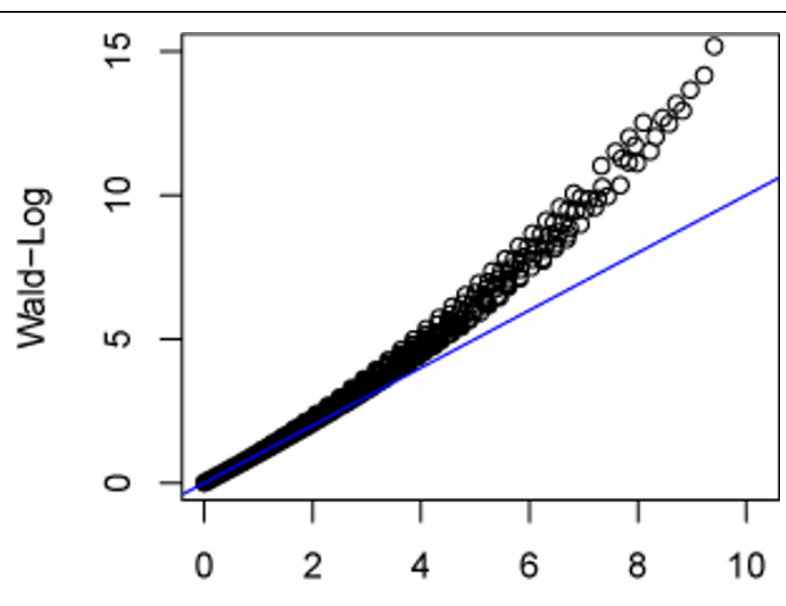

LRT

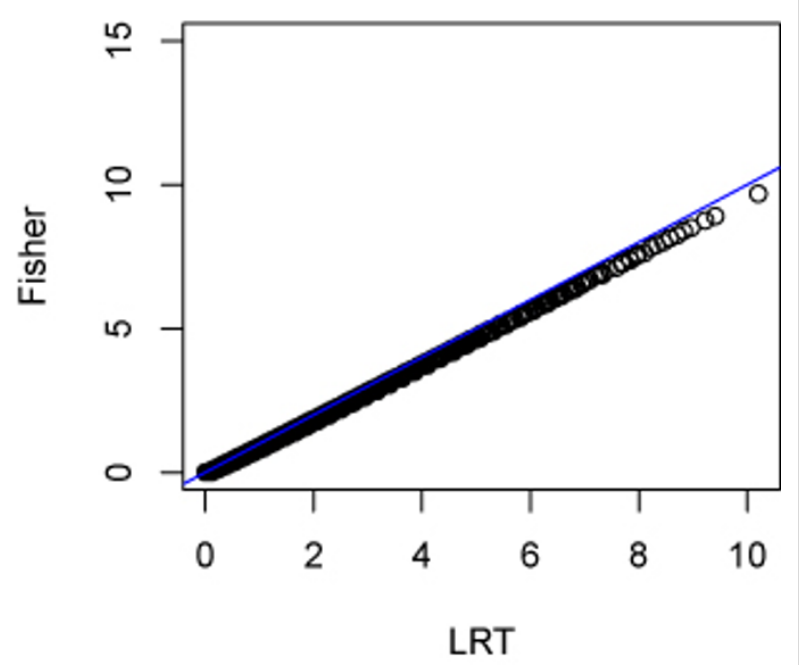

Figure 4 P-values of the $\mathbf{2 6 5 7}$ low espression genes from different methods. Compare p-values (-log10 scale) obtained from other methods with those from LRT for the 2657 low expression genes whose average counts per lane are between 1 and 10 for each sample.

LRT has been used by Marioni et al [3] based on a GLM, where they first normalize the data across lanes by a global scale normalization method so that the total counts on each lane are the same after normalization.

\section{Conditional binomial test}

It can be shown that the conditional distribution of the sum of $\mathrm{X}_{1 \mathrm{j}}$ given the total sum of $\mathrm{X}_{\mathrm{ij}}$ has a binomial distribution $[10,16]$. More specifically,

$$
\sum_{j=1}^{n_{1}} X_{1 j} \mid \sum_{j=1}^{n_{1}} X_{1 j}+\sum_{j=1}^{n_{2}} X_{2 j}=k \sim \operatorname{Bin}(k, p), \quad \text { where } \quad \mathrm{p}=\frac{n_{1} \lambda_{1}}{n_{1} \lambda+n_{2} \lambda_{2}}
$$

Under the null hypothesis, $p_{0}=\frac{n_{1}}{\eta_{1}+n_{2}}$ and the exact $\mathrm{p}$-value can be calculated from the binomial distribution $\operatorname{Bin}\left(\mathrm{n}_{1}+\mathrm{n}_{2}, \mathrm{p}_{0}\right)$.

\section{Wald test}

The Wald test statistic is [11]:

$$
T_{\text {Wald }}=\frac{\sum_{j=1}^{n_{1}} X_{1 j}-\frac{n_{1}}{n_{2}} \sum_{j=1}^{n_{2}} X_{2 j}}{\sqrt{\frac{n_{1}}{n_{2}}\left(\sum_{j=1}^{n_{1}} X_{1 j}+\sum_{j=1}^{n_{2}} X_{2 j}\right)}} .
$$



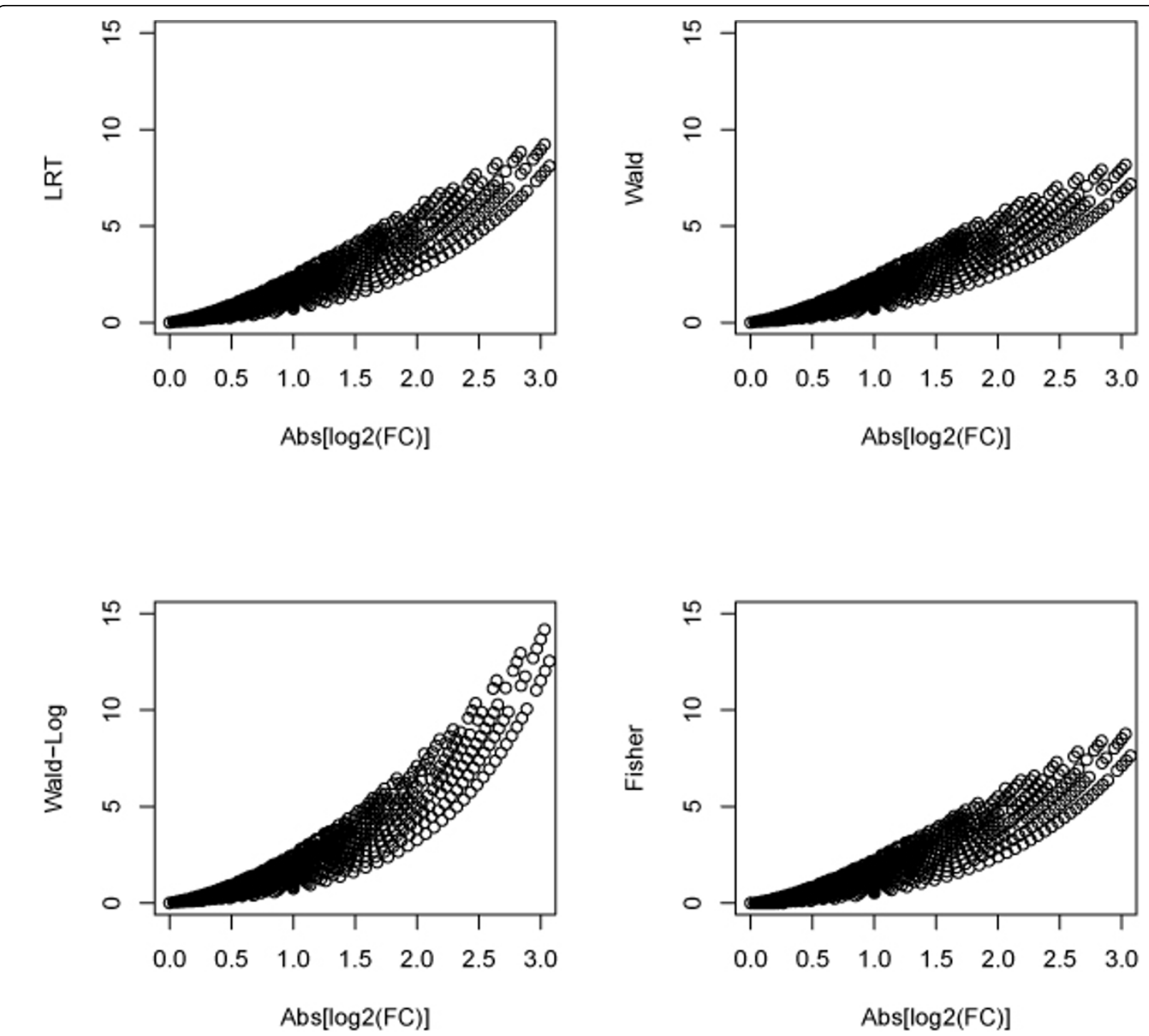

Figure 5 P-values vs. $\log 2(\mathrm{FC})$ of the 2657 low espression genes from different methods. P-value (-log 10 scale) obtained by various methods vs. fold change (log2 scale) for the 2657 low expression genes whose average counts per lane are between 1 and 10 for each sample.

Under the null hypothesis, $\mathrm{T}_{\text {Wald }}$ has an asymptotic standard normal distribution.

\section{Wald test for logarithm transformed data (Wald-Log)}

The Wald test can be also applied to the logarithm transformed count data [11]. We call it Wald-Log test. The test statistic can be derived by using Delta method and has the following form:

$$
T_{\text {Wald }- \text { Log }}=\frac{\log \left[\left(\sum_{j=1}^{n_{1}} X_{1 j} / n_{1}\right) /\left(\sum_{j=1}^{n_{2}} X_{2 j} / n_{2}\right)\right]}{\sqrt{\frac{2+n_{1} / n_{2}+n_{2} / n_{1}}{\sum_{j=1}^{n_{1}} X_{1 j}+\sum_{j=1}^{n_{2}} X_{2 j}}}}=\frac{\log (\text { Fold Change })}{\sqrt{\frac{2+n_{1} / n_{2}+n_{2} / n_{1}}{\sum_{j=1}^{n_{1}} X_{1 j}+\sum_{j=1}^{n_{2}} X_{2 j}}}} .
$$

Variance stabilizing transformation test (VST)

The VST statistic [10,13] has the form:

$$
T_{V S T}=2\left[\sqrt{\sum_{j=1}^{n_{1}} X_{1 j}+3 / 8}-\sqrt{\left(\sum_{j=1}^{n_{2}} X_{2 j}+3 / 8\right) n_{1} / n_{2}}\right] / \sqrt{1+n_{1} / n_{2}} .
$$

\section{Fisher exact test}

Fisher exact test is applied to the following 2 by 2 table (Table 4):

The exact $\mathrm{p}$-value is then calculated based on the conditional hypergeometric distribution. 


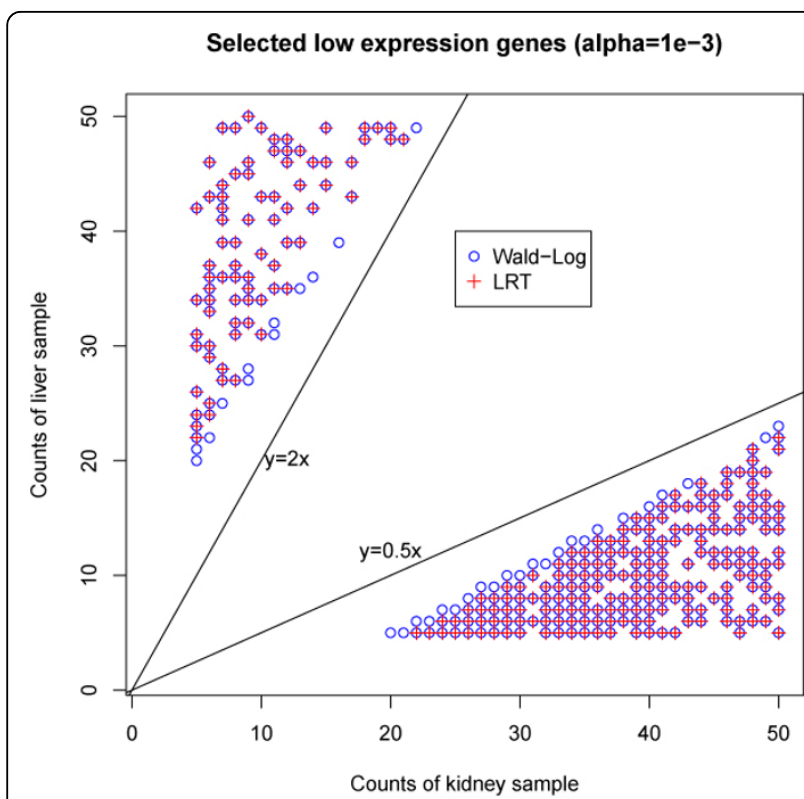

Figure 6 Counts under two conditions for each of the selected low expression genes. With significance level $10^{-3}$, the Wald-Log test selects 581 out of 2657 low expression genes. While the LRT selects 498 of the 581 genes. The count for each selected gene of the two samples is plotted.

Table 3 Numbers of DEGs of low expression obtained by various methods.

\begin{tabular}{lllllll}
\hline Cutoff & LRT & Cond & Wald & Wald-Log & VST & Fisher \\
\hline 1e-3 & 498 & 434 & 462 & 581 & 498 & 434 \\
$1 \mathrm{e}-4$ & 303 & 258 & 270 & 403 & 305 & 260 \\
$1 \mathrm{e}-5$ & 195 & 166 & 166 & 275 & 201 & 166 \\
$1 \mathrm{e}-6$ & 113 & 86 & 84 & 209 & 119 & 86 \\
\hline
\end{tabular}

Numbers of DEGs selected from the 2657 low expression genes with different cutoff $p$-values by each method.

Table 4 The 2 by 2 table for Fisher test.

\begin{tabular}{lll}
\hline Condition 1 & $\sum_{j=1}^{n_{1}} x_{1 j}$ & $\sum_{j=1}^{n_{1}} m_{1 j}-\sum_{j=1}^{n_{1}} x_{1 j}$ \\
\hline Condition 2 & $\sum_{j=1}^{n_{2}} x_{2 j}$ & $\sum_{j=1}^{n_{2}} m_{2 j}-\sum_{j=1}^{n_{2}} x_{2 j}$
\end{tabular}

The 2 by 2 table for Fisher test (where $m_{i j}$ is the total number of counts on lane ij).

\section{Acknowledgements}

ZC would like to thank the support from the NIH grant (UL1 RRO24148), awarded to the University of Texas Health Science Center at Houston. This article has been published as part of BMC Systems Biology Volume 5 Supplement 3, 2011: BIOCOMP 2010 - The 2010 International Conference on Bioinformatics \& Computational Biology: Systems Biology. The full contents of the supplement are available online at http://www.biomedcentral.com/ $1752-0509 / 5$ ? issue $=\$ 3$.

\section{Author details}

${ }^{1}$ Biostatistics Epidemiology Research Design Core, Center for Clinical and Translational Sciences, The University of Texas Health Science Center at Houston, Houston, TX 77030, USA. ${ }^{2}$ The Chem 21 Group Inc, 1780 Wilson Drive, Lake Forest, IL 60045, USA. ${ }^{3}$ Department of Statistical Science, Southern Methodist University, Dallas, TX 75275, USA. ${ }^{4}$ School of Mathematics, University of Manchester, Manchester, M13 9PL, UK. ${ }^{5}$ Rush University Cancer Center, Rush University Medical Center, Chicago, IL 60612 USA. ${ }^{6} 6$ Department of General Surgery and Immunology and Microbiology, Rush University Medical Center, Chicago, IL 60612, USA. ${ }^{7}$ Department of Radiation Oncology Massachusetts General Hospital and Harvard Medical School Boston, MA 02114, USA. ${ }^{8}$ Department of Internal Medicine and Biochemistry, Rush University Medical Center, Chicago, IL 60612, USA.

\section{Authors' contributions}

$\mathrm{C}$ designed the algorithm, conducted the study, and drafted the manuscript; $J \mathrm{~L}$ analyzed the data and assisted in programming; YD coordinated and directed the whole project. HKTN, SN, HLK, JYY and YD participated in the analysis and discussion. All authors read and approved the final manuscript.

\section{Competing interests}

The authors declare that they have no competing interests.

Published: 23 December 2011

\section{References}

1. Bullard JH, Purdom E, Hansen KD, Dudoit S: Evaluation of statistical methods for normalization and differential expression in mRNA-Seq experiments. BMC Bioinformatics 2010, 11:94.

2. Ingolia NT, Ghaemmaghami S, Newman JR, Weissman JS: Genome-wide analysis in vivo of translation with nucleotide resolution using ribosome profiling. Science 2009, 324(5924):218-223.

3. Marioni JC, Mason CE, Mane SM, Stephens M, Gilad Y: RNA-seq: an assessment of technical reproducibility and comparison with gene expression arrays. Genome Res 2008, 18(9):1509-1517.

4. Wang Z, Gerstein M, Snyder M: RNA-Seq: a revolutionary tool for transcriptomics. Nat Rev Genet 2009, 10(1):57-63.

5. Robinson MD, Oshlack A: A scaling normalization method for differential expression analysis of RNA-seq data. Genome Biol 2010, 11(3):R25.

6. Bloom JS, Khan Z, Kruglyak L, Singh M, Caudy AA: Measuring differential gene expression by short read sequencing: quantitative comparison to 2-channel gene expression microarrays. BMC Genomics 2009, 10:221.

7. Wang L, Feng Z, Wang X, Zhang X: DEGseq: an R package for identifying differentially expressed genes from RNA-seq data. Bioinformatics 2010, 26(1):136-138.

8. Robinson MD, Smyth GK: Moderated statistical tests for assessing differences in tag abundance. Bioinformatics 2007, 23(21):2881-2887.

9. Wu Z, Jenkins BD, Rynearson TA, Dyhrman ST, Saito MA, Mercier M, Whitney LP: Empirical bayes analysis of sequencing-based transcriptional profiling without replicates. BMC Bioinformatics 2010, 11:564.

10. Gu K, Ng HK, Tang ML, Schucany WR: Testing the ratio of two poisson rates. Biom J 2008, 50(2):283-298.

11. Ng HK, Tang ML: Testing the equality of two Poisson means using the rate ratio. Stat Med 2005, 24(6):955-965.

12. Ng HKT, Gu K, Tang ML: A comparative study of tests for the difference of two Poisson means. Computational Statistics and Data Analysis 2007, 51:3085-3099

13. Huffman MD: An improved approximate two-sample Poisson test. Applied Statistics 1984, 33:224-226.

14. Oshlack A, Wakefield MJ: Transcript length bias in RNA-seq data confounds systems biology. Biol Direct 2009, 4:14.

15. Young MD, Wakefield MJ, Smyth GK, Oshlack A: Gene ontology analysis for RNA-seq: accounting for selection bias. Genome Biol 2010, 11(2):R14.

16. Przyborowski J, Wilenski H: Homogeneity of results in testing samples from Poisson series. Biometrika 1940, 31:313-323.

doi:10.1186/1752-0509-5-S3-S1

Cite this article as: Chen et al:: Statistical methods on detecting differentially expressed genes for RNA-seq data. BMC Systems Biology 2011 5(Suppl 3):S1. 